\title{
Erratum: Full spin flipping in the presence of full Siberian Snake [Phys. Rev. ST Accel. Beams 11, 091001 (2008)]
}

\author{
M. Bai and T. Roser \\ (Received 7 January 2009; published 26 January 2009) \\ PACS numbers: 29.27.Hj, 99.10.Cd
}

DOI: $10.1103 /$ PhysRevSTAB.12.019901

It was brought to our attention by Sateesh Mane that the plot in Fig. 5 did not match its caption in the original paper. We also corrected a typo in the caption. Please see the correct Fig. 5 below.

We apologize for the mistake. The conclusions of the paper are unchanged.

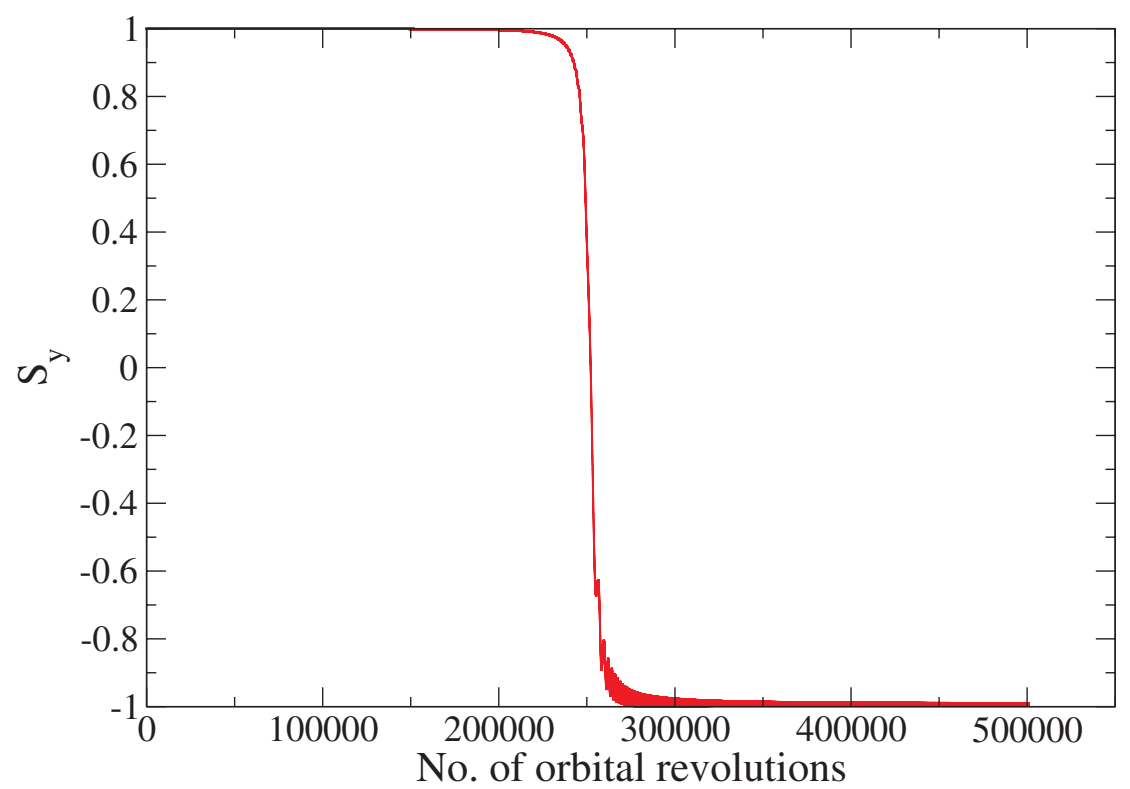

FIG. 5. (Color) This plot shows the vertical component of the spin vector as a function of number of orbital turns for a simulation done using SPINK with the RHIC lattice with all the quadrupoles included. The layout of the spin flipper setup is similar to what is shown in Fig. 3. Instead of a $\phi_{0}=90^{\circ}$ spin rotator in between the two rf dipoles, a $\phi_{0}=30^{\circ}$ spin rotator is used for this tracking and the amplitude of the spin rotation of the two rf dipoles $\tilde{\phi}_{\text {osc }}$ was increased to 2.28 mrad with a phase difference of $\chi_{1}-\chi_{2}=210^{\circ}$. 